\title{
1 Resource limitation modulates the fate of dissimilated nitrogen in a dual-pathway
}

2 Actinobacterium

3 David C. Vuono ${ }^{1,6}$, Robert W. Read ${ }^{1}$, James Hemp ${ }^{2}$, Benjamin W. Sullivan ${ }^{3}$, John A. Arnone

$4 \quad$ III $^{1}$, Iva Neveux ${ }^{1}$, Bob Blank $^{4}$, Carl Staub ${ }^{5}$, Evan Loney ${ }^{1}$, David Miceli ${ }^{1}$, Mari Winkler $^{6}$, Romy

5 Chakraborty $^{7}$, David A. Stahl ${ }^{6}$, Joseph J. Grzymski ${ }^{1, *}$

$6{ }^{1}$ Division of Earth and Ecosystem Sciences, Desert Research Institute, Reno, NV 89512, USA

$7 \quad{ }^{2}$ Division of Geological and Planetary Sciences, California Institute of Technology, Pasadena,

8 CA, USA

$9{ }^{3}$ Department of Natural Resources and Environmental Science, University of Nevada, Reno, NV

10 89557, USA

$11{ }^{4}$ Agricultural Research Service, U.S. Department of Agriculture, Reno, NV, 89512, USA

$12{ }^{5}$ Agtron, Inc. 9395 Double R Blvd, Reno, NV 89521, USA

$13{ }^{6}$ Department of Civil and Environmental Engineering, University of Washington, Seattle, WA, 1498195, USA

$15 \quad{ }^{7}$ Lawrence Berkeley National Laboratory, Berkeley, CA, 94729, USA

$16{ }^{*}$ Correspondence: Joseph J. Grzymski, Division of Earth and Ecosystem Sciences, Desert

17 Research Institute, Reno, 2215 Raggio Parkway, NV 89512, USA

\section{Email: Joe.Grzymski@dri.edu}




\section{Abstract}

Respiratory ammonification and denitrification are two evolutionarily unrelated dissimilatory nitrogen $(\mathrm{N})$ processes central to the global $\mathrm{N}$ cycle, the activity of which is thought to be controlled by carbon (C) to nitrate $\left(\mathrm{NO}_{3}{ }^{-}\right)$ratio. Here we find that Intrasporangium calvum $\mathrm{C} 5$, a novel menaquinone-based dual-pathway denitrifier/respiratory ammonifier, disproportionately utilizes ammonification rather than denitrification when grown under carbon or nitrate limitation, not $\mathrm{C}: \mathrm{NO}_{3}{ }^{-}$ratio. Instead, $\mathrm{C}: \mathrm{NO}_{3}{ }^{-}$ratio is a confounding variable for resource limitation. We find that the protein atomic composition for denitrification modules (NirK) are significantly cost minimized for $\mathrm{C}$ and $\mathrm{N}$ compared to ammonification modules (NrfA), indicating that resource limitation is a major selective pressure imprinted in the architecture of these proteins. The evolutionary precedent for these findings suggests ecological and biogeochemical importance as evidenced by higher growth rates when I. calvum grows predominantly using its ammonification pathway and by assimilating its end-product (ammonium) for growth under ammonium-deplete conditions. Genomic analysis of I. calvum further reveals a versatile ecophysiology to cope with nutrient stress and redox conditions. Metabolite and transcriptional profiles during growth indicate that transcript abundances encoding for its nitrite reducing enzyme modules, $\mathrm{NrfAH}$ and NirK, significantly increase in response to nitrite production. Mechanistically, our results suggest that pathway selection is driven by intracellular redox potential (redox poise), which may be lowered during resource limitation, thereby decreasing catalytic activity of upstream electron transport steps needed for denitrification enzymes. Our work advances our understanding of the biogeochemical flexibility of N-cycling organisms, pathway evolution, and ecological foodwebs.

\section{Introduction}

42 Globally, respiratory ammonification and denitrification are vital nitrogen $(\mathrm{N})$ dissimilation

43 pathways that either retain reactive $\mathrm{N}$ to support net primary productivity or close the $\mathrm{N}$-cycle through the release of gaseous $\mathrm{N}$, respectively [1]. The environmental controls on these two

45 pathways, particularly the ratio of electron-donor to electron-acceptor (e.g., $\mathrm{C}: \mathrm{NO}_{3}{ }^{-}$) [2], have

46 gained attention [3-7] due to increased anthropogenic $\mathrm{N}$ inputs into the environment [8].

47 However, the effects of resource limitation on growth and pathway selection (i.e., allocation of C 
and $\mathrm{N}$ to dissimilatory and assimilatory processes), which are often confounded by $\mathrm{C}: \mathrm{NO}_{3}{ }^{-}$ratio, have not been tested. Strong selective pressures from Earth's shifting biogeochemistry and oxidation-state have driven evolutionary adaptions to microbial electron transport chains (ETC) $[9,10]$, respiratory chain redox potentials [11-13], and protein atomic composition $[14,15]$, may shed light on how these pathways are regulated in contemporary organisms. Here, by identifying the biochemical and evolutionary differences between respiratory ammonification and denitrification, we disentangle the ecological significance and molecular mechanisms of electron transfer through either pathway in a dual pathway organism.

56 From a biochemical standpoint, the primary difference between respiratory ammonification and

57 denitrification is their respective source of reducing equivalents in the ETC: 1) heme-based

58 cytochrome c nitrite reductase used in respiratory ammonification receive electrons directly from

59 the quinone (Q) pool [16] while 2) copper and $c d_{1}$ nitrite reductases used in denitrification

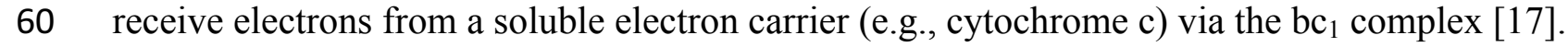

61 From an evolutionary standpoint, we can place each N-module's origin to a putative time in

62 Earth history based on the metal co-factors that would have been bioavailable: heme-based

63 cytochromes in an ancient, more reduced, environment compared to the copper-containing nitrite

64 reductases in an oxidizing environment [18]. The bioenergetic chains of microorganisms also

65 underwent selective pressure to shift from low-potential (LP) to high-potential (HP) quinones in 66 response to Earth's oxygenation $[11,12]$. Menaquinone (MK) is thought to be the ancestral type

67 of LP quinone [19]. Organisms that use ubiquinone (UQ) are thought to have evolved under high

$68 \mathrm{O}_{2}$ tensions with $\alpha-, \beta$-, $\gamma$-proteobacteria as the only bacterial clades to use UQ [12]. Surprisingly, 69 our understanding for the biochemistry of denitrification is based predominantly on HP UQ70 based systems [20], leaving a significant knowledge gap in the physiology and biochemistry of

71 LP MK-based denitrifiers and how they link electron transfer with energy capture under resource 72 limitation [21-23].

73 In order to resolve the mechanisms of $\mathrm{C}: \mathrm{NO}_{3}{ }^{-}$control on pathway selection and better understand 74 branched respiratory chains in LP-based nitrate-reducing organisms, we undertook the 75 characterization of the novel Gram-positive Actinobacterium strain Intrasporangium calvum C5: 76 a dual-pathway nitrite reducer that uses MK as sole pool quinone. Here we show that over a 77 range of $\mathrm{C}: \mathrm{NO}_{3}{ }^{-}$ratios, duplicated at two substrate concentrations, I. calvum disproportionately 
utilizes its ammonia-forming pathway during $\mathrm{C}$ limitation $\left(\leq 0.4 \mathrm{mM}\right.$ lactate), when $\mathrm{C}: \mathrm{NO}_{3}{ }^{-}{ }^{-}$ratios are $<1$ (an observation contrary to the current paradigm). Using a genome-guided approach coupled to time-series transcriptomics and metabolite profiles, we identified differentially expressed genes in the bacterium's ETC and central metabolic pathways. Using this information to inform a metabolic reconstruction of the ETC and extensive literature on the biochemistry of the $b_{1}$ complex, we propose a new mechanism by which these two pathways are regulated at the biochemical level.

\section{Materials and Methods}

\section{Culture Conditions}

87 Media preparation: All cultures were grown at $30^{\circ} \mathrm{C}$ and shaken at $250 \mathrm{rpm}$. Nitrate reducing

88 minimal media was prepared with the following final concentrations: $\mathrm{NaCl}(0.6 \mathrm{mM}), \mathrm{NH}_{4} \mathrm{Cl}$

$89(1.75 \mathrm{mM})$ (for ammonium replete conditions but not used in NH4-deplete conditions), $\mathrm{MgCl} 2$

$90(0.2 \mathrm{mM}), \mathrm{CaCl} 2(0.04 \mathrm{mM}), \mathrm{KCl}(0.1 \mathrm{mM}), \mathrm{K} 2 \mathrm{HPO} 4(0.01 \mathrm{mM}), \mathrm{NaHCO}-(0.3 \mathrm{mM})$, cysteine

$91(1 \mathrm{mM})$ as reducing agent, resazurin as redox indicator, and trace elements and trace vitamin

92 solutions as reported $[24,25]$. $1 \mathrm{M}$ sterile filtered $(0.2 \mu \mathrm{m})$ Concentrated stocks of $60 \% \mathrm{w} / \mathrm{w}$

93 sodium DL-lactate solution (Sigma-Aldrich, St. Louis, MO, USA), sodium-nitrate and sodium-

94 nitrite ( $\geq 99 \%$, Fisher Scientific, Pittsburg, PA, USA) were diluted into media prior to

95 autoclaving to achieve the desired ${\mathrm{C}: \mathrm{NO}_{3}}^{-}$ratio. $\mathrm{C}: \mathrm{NO}_{3}{ }^{-}$ratio was calculated based on [3] where

96 the number of $\mathrm{C}$ atoms (n) in the e-donor is multiplied by the concentration of the e-donor,

97 divided by the number of $\mathrm{N}$ atoms in the e-acceptor multiplied by the concentration of the e-

98 acceptor (Table S4). See SI Materials and Methods for complete description of Hungate

99 technique prepared media. Mean $\mathrm{pH}$ for all culture vessels (time series and end-point; Table S5),

100 measured at the end of each experiment, was $7.3 \pm 0.05(n=144)$.

\section{Analytical procedures}

102 Growth Curve/Cell counts/Yield Measurements: Growth curves were measured from scratch103 free Balch-tubes grown cultures using an automated optical density reader at $\mathrm{OD}_{600} \mathrm{~nm}$ 104 (Lumenautix LLC, Reno, NV). End-point cultures were monitored until all replicates reached 105 stationary phase (65-100 hours depending on ${\mathrm{C}: \mathrm{NO}_{3}}^{-}$treatment) (Figure S6). Cell counts were 106 performed by fixing cells in $4 \%$ paraformaldehyde (final concentration) for 20 minutes, filtered 
onto $0.2 \mu \mathrm{m}$ pore-sized black polycarbonate filters. A complete description is provided in SI Materials and Methods. Biomass concentrations were measured by filtration and drying as per standard protocol [26]. A complete description is provided in SI Materials and Methods.

Ion and Gas Chromatography Measurements: A dual channel Dionex ICS-5000+ (Thermo Scientific) ion chromatograph (IC) was used to measure organic (lactate, acetate, and formate) and inorganic (nitrite and nitrate) anions on an AS11-HC column and cations (ammonium) on a CS-16 column from the bacterial growth media. A complete description is provided in SI Materials and Methods.

\section{Phylogenetic, Genomic, and Transcriptomic Analysis}

Genomic DNA was assembled using Canu (version 1.7.1) with an estimated genome size of 5 million base pairs [27]. The resulting single contiguous fragment was aligned to the $I$. calvum 7KIP genome (Acc: NC_014830.1) to compare sequence similarity in Mauve[28, 29]. Genome annotation for C5 was performed through the NCBI Prokaryotic Genome pipeline (www.ncbi.nlm.nih.gov/genome/annotation_prok/). Additional gene prediction analysis and functional annotation was performed by the DOE Joint Genome Institute (JGI) using the Isolate Genome Gene Calling method (Prodigal V2.6.3 February, 2016) under the submission ID 172966. The complete genome sequence and annotation is available in the NCBI database under the BioProject number PRJNA475609. A complete description of the phylogenetic, pathway analysis, and cost-minimization calculations is provided in SI Material and Methods. For transcriptomic analysis, the resulting raw reads were inspected using FastQC [30] to determine quality, read length, and ambiguous read percentage. Reads were trimmed based on quality score with a sliding window of 5 base pairs, quality cutoff of 28 , trailing cutoff quality score of 10 , as well as adapter contamination removal in Trimmomatic [31]. A complete description is provided in SI Materials and Methods. Statistical analyses were conducted in the R environment for statistical computing (r-project.org). Data that was tested using parametric statistical analysis were first validated for normality by visualizing the data as a histogram and testing via ShapiroWilks test for normality.

\section{Results}


136 Genomic analysis of I. calvum C5. We sequenced and analyzed the genome of I. calvum C5 to 137 first compare its similarity to the type species I. calvum 7KIP. We identified a high degree of sequence similarity to 7KIP based on three homologous sequence regions as locally collinear blocks (SI Results). Genome size of C5 was 4,025,044 base pairs (bp), only 662 bp longer than 7KIP. Genomic analysis of the ETC revealed the typical suite of complexes common to alternative $\mathrm{NDH}-2 \mathrm{NADH}$ dehydrogenase, cytochrome $\mathrm{bc}_{1}$ complex, high-oxygen adapted cytochrome c oxidase (A-family), and low-oxygen adapted cytochrome $b d$ oxidase. The $\mathrm{bc}_{1}$ complex subunits are also located immediately upstream of cytochrome c oxidase, suggesting that these enzymes are encoded in a single operon creating a supercomplex. Despite I. calvum's seeming propensity for aerobic growth on a number of growth media [32], its bioenergetic system uses MK as its sole pool quinone. I. calvum also possesses multiple pathways for supplying electrons into the MK-pool, such as formate, malate, hydroxybutyrate, and glycerophosphate dehydrogenases. Once in the MK-pool, there are alternative pathways for $\mathrm{MKH}_{2}$ oxidation that can circumvent the $\mathrm{bc}_{1}$ complex, such as a membrane-bound respiratory nitrate reductase module (NarG). In addition, to $\mathrm{NarG}$, its dissimilatory $\mathrm{N}$ module composition consists of a truncated denitrification pathway $\left(\mathrm{N}_{2} \mathrm{O}\right.$ is a terminal product) using a copper nitrite reductase NirK and quinol-dependent nitric oxide reductase qNor. I. calvum also possesses both catalytic and membrane anchor subunits (NrfA and $\mathrm{NrfH}$, respectively) for a pentaheme cytochrome c module involved in respiratory nitrite ammonification.

\section{I. calvum encodes for a functional $\mathrm{NrfAH}$ complex and assimilates $\mathrm{NH}_{4}{ }^{+}$via respiratory} nitrite ammonification. To gain insight into possible function of the NrfAH complex, we aligned the NrfA protein sequences from C5 and 7KIP to a collection of 33 recognized cytochrome c nitrite reductases from published annotated genomes (Table S1). This confirmed that NrfA from I. calvum is a member of the $\mathrm{CxxCH} 1^{\text {st }}$ heme motif group (Figure 1A), which forms one of four clades on the NrfA phylogenetic tree. We then queried the genomes of the taxa in our phylogeny for other annotated N-reducing modules used in nitrate reduction, nitrite reduction, NO-forming nitrite reduction, and primary pool quinone. Among the three major clades of NrfA, at least 5 additional taxa are noted having dissimilatory N-module inventories containing dual respiratory pathways: $S$. thermophilum, B. azotoformans, B. bataviensis, B. bacteriovorus, and Candidatus N. inopinata, (Figure 1A). None of the taxa in our NrfA 
phylogeny harbored the $c d_{1}$ nitrite reductases (NirS). Due to the exclusive NirK representation in dual-pathway membership, we asked whether there might be differences in protein atomic composition between NirK and NrfA, given the disparate evolutionary origins of these modules [33]. We collected 20 additional publicly available NirK protein sequences from nondualpathway denitrifiers (Table S1) and calculated the protein $\mathrm{C}$ and $\mathrm{N}$ composition for our

172 NirK/NrfA collection as atoms per residue side-chain (Figure 1B). These results showed a

173 significant depletion in $\mathrm{C}$ and $\mathrm{N}$ atoms per residue side-chain (ARSC) for NirK compared to

$174 \operatorname{NrfA}(\mathrm{C}$ and N: $p<0.001$; t-test), indicating that resource constraints are imprinted on the 175 evolution of these proteins.

176 We next tested the functionality of I. calvum's Nrf complex by growing the bacterium under 177 reducing conditions ( $8 \mathrm{mM}$ lactate, $12 \mathrm{mM}$ nitrate, ammonium-replete). We then performed a

178 state-transition where biomass from late-exponential growth phase was collected and 179 anaerobically inoculated into ammonia-deplete media (Figure 1C; SI Results). Despite no 180 detectable amounts of ammonium produced in the media over time, cell counts increased $1815.4 \times 10^{5} \pm 8.9 \times 10^{4}$ cells $/ \mathrm{mL}\left(0.126 \pm 0.02\right.$ optical absorbance at $\left.\mathrm{OD}_{600}\right)$ over a 48 -hour incubation, 182 indicating consumption of ammonium produced by NrfA. Net ammonium production was $18313 \pm 2.7 \mu$ moles with the remainder of dissimilated $\mathrm{N}$ being used by the denitrification pathway $184\left(24 \pm 4.2 \mu\right.$ moles $\left.\mathrm{N}_{2} \mathrm{O}-\mathrm{N}\right)$, resulting in a recovery of $97.4 \%$ dissimilated $\mathrm{N}$. These results confirmed that I. calvum $\mathrm{C} 5$ has a functional Nrf complex and also consumes the product (ammonium) of respiratory nitrite ammonification.

\section{Respiratory nitrite ammonification exceeds denitrification under C-limitation. We}

investigated $\mathrm{C}: \mathrm{NO}_{3}{ }^{-}$control on respiratory ammonification versus denitrification on cultures of $I$. calvum $\mathrm{C} 5$ over a high resource $\mathrm{C}: \mathrm{NO}_{3}{ }^{-}$range (16-0.4 mM lactate, $12 \mathrm{mM}$ nitrate; ratio 4-0.1) and low resource $\mathrm{C}: \mathrm{NO}_{3}{ }^{-}$range (1.6-0.04 mM lactate, $1.2 \mathrm{mM}$ nitrate; ratio 4-0.1). This experimental design enabled us to evaluate $\mathrm{C}: \mathrm{NO}_{3}{ }^{-}$control over a broader range than previous studies that only considered ratios $\geq 1.5[3,4,34]$, while also testing the effects of resource concentration on pathway selection. Under all the treatments tested, gas and ion chromatography measurements showed products of both respiratory pathways, differing only in the relative

195 fraction of $\mathrm{N}_{2} \mathrm{O}$ versus ammonium production across treatments (Figure 2). At high resource concentrations, respiratory ammonification did not prevail at high $\mathrm{C}: \mathrm{NO}_{3}{ }^{-}$ratios (Figure $2 \mathrm{~A}$, 
Figure 2B, left panels; Table S2). Instead, significantly greater amounts of $\mathrm{N}_{2} \mathrm{O}$ were produced over ammonium, though nitrite was still the major extracellular end-product of nitrate respiration. Despite the predominance of $\mathrm{N}_{2} \mathrm{O}$ production under the high resource concentrations, ammonium production exceeded $\mathrm{N}_{2} \mathrm{O}$ production only at the lowest $\mathrm{C}: \mathrm{NO}_{3}{ }^{-}$ratio $(0.4 \mathrm{mM}$ lactate, ratio $=0.1$ ) (Figure 2) and accounted for $76.2 \pm 0.1 \%$ of dissimilated $\mathrm{N}$.

Results from the low resource dataset provided weak support for the strict stoichiometry hypothesis that $\mathrm{C}: \mathrm{NO}_{3}{ }^{-}$controls pathway selection. Ammonia exceeded $\mathrm{N}_{2} \mathrm{O}$ production only under one high $\mathrm{C}: \mathrm{NO}_{3}{ }^{-}$ratio treatment (ratio $=4 ; 1.6 \mathrm{mM}$ lactate; Figure 2A, Figure 2B, right panels). However, at ratios $\leq 1\left(\leq 0.4 \mathrm{mM}\right.$ lactate), significantly more ammonium than $\mathrm{N}_{2} \mathrm{O}$ was produced. On average, respiratory ammonification accounted for $78.1 \pm 8.9 \%$ of dissimilated $\mathrm{N}$ for lactate concentrations $\leq 0.4 \mathrm{mM}$. When these results are taken in context with cell physiology, we observed a significant and positive relationship between specific growth rate $(\mu)$ and the fraction of $\mathrm{N}$ dissimilated by respiratory ammonification $\left(R^{2}=0.5 ; p<0.001\right)$ (Figure $2 \mathrm{C}, \mathrm{S} 1$; Table S3).

\section{Resource concentration influences the metabolite profiles of ammonium and $\mathrm{N}_{2} \mathrm{O}$}

212 production. Given the co-occurrence of end products from both pathways during the end-point experiments (Figure 2), we next investigated the timing of ammonium and $\mathrm{N}_{2} \mathrm{O}$ production

214 relative to metabolite profiles for lactate, nitrate/nitrite, and growth phase at two resource

215 concentrations with the same ratio ( $8 \mathrm{mM}$ and $0.8 \mathrm{mM}$ lactate, ratio=2; Figure 1A, Figure $\mathrm{S} 2$,

216 Figure S3). Despite ample e-donor and e-acceptor available for growth, the high resource

217 cultures entered a quasi-stationary phase at $\sim 50$ hours, after which there was continued slow

218 growth (Figure 1A). Metabolite profiles showed that ammonium and $\mathrm{N}_{2} \mathrm{O}$ production began

219 simultaneously, as soon as nitrite was produced from nitrate reduction. The low resource cultures entered stationary phase at $\sim 40$ hours (Figure S2) after nitrate had been fully utilized. No further

221 cell growth was observed after stationary phase was reached. These results show that cell growth

222 occurred primarily on the reduction of nitrate, while nitrite reduction to ammonium and $\mathrm{N}_{2} \mathrm{O}$

223 occurred during a stationary growth phase, demonstrating that microbial activity is not always

224 correlated with growth. The metabolite profiles for ammonium and $\mathrm{N}_{2} \mathrm{O}$ at low resources (Figure

$225 \mathrm{~S} 2$ ) did not mirror those observed at high resources (Figure 2A). The rate of $\mathrm{N}_{2} \mathrm{O}$ production

226 significantly decreased and ammonium production oscillated rather than steadily increase 
227 through time. These differences in metabolite profiles, further demonstrate that concentration

228 influences the activities of pathway bifurcation. Repeated time series experiments that were

229 extended up to 300 hours show that nitrite is slowly depleted, but does not get fully consumed

230 (Figure S3). When cultures were given nitrite, instead of nitrate as a terminal electron acceptor (8 $231 \mathrm{mM}$ lactate, $12 \mathrm{mM}$ nitrite; ratio=2), we observed no immediate growth (as was observed with

232 nitrate) but measured more $\mathrm{N}_{2} \mathrm{O}$ than ammonium production $\left(33.4 \pm 4.8 \mu\right.$ moles $\mathrm{N}_{2} \mathrm{O}-\mathrm{N}$ and

$2338.0 \pm 2.5 \mu$ moles $\mathrm{NH}_{4}{ }^{+}$, respectively) (Figure $\mathrm{S} 4$ ), demonstrating respiratory ammonification does

234 not exceed denitrification when nitrite is supplied as the sole acceptor in I. calvum.

235 Nitrite-reducing modules are up-regulated during late exponential- and stationary-phase

236 growth. In order to gain insight into mechanisms of gene regulation and transcriptional

237 organization of I. calvum, we conducted RNA-Seq in parallel with the high resource time-series

238 metabolite profile (Figure 3A). This approach enabled us to compare genome-wide differential 239 expression based on $\log _{2}$ fold change (lfc) of RNA extracted from three growth phases: early 240 exponential (EE), late exponential (LE), and stationary (ST) (Figure 3B, Figure S5). Within the 241 central metabolic pathway beginning with the conversion of lactate to pyruvate, we observed a 242 moderate decrease in transcript abundance of L-lactate dehydrogenase (LDH) (Intca 16740)

243 between EE-LE and -ST (lfc $=-1.6 \pm 0.7 ;-1.9 \pm 0.7$ ), respectively. Lactate utilization protein C

244 (LUP) (Intca_04080), an enzyme involved in lactate degradation, also showed a moderate and 245 significant decrease in transcript abundance between EE-LE and -ST (1fc $=-1.6 \pm 0.6 ;-2.4 \pm 0.6$, $246 p=0.002$ ), respectively. I. calvum encodes for two parallel metabolic pathways for pyruvate 247 conversion to acetyl-CoA: pyruvate dehydrogenase (PDH) (Intca_01255) and pyruvate 248 ferredoxin oxidoreductase (PFOR) (Intca_15510). For PDH, there was a significant and 249 moderate increase in transcript abundance between EE-LE and -ST (lfc $=2.1 \pm 0.6, p=0.002$;

$2501.5 \pm 0.6)$, respectively. For PFOR, there was a minor decrease in transcript abundance between 251 EE-LE $(1 \mathrm{fc}=-0.43 \pm 0.5)$, and then a moderate increase in transcript abundance between EE-ST 252 (1.1 \pm 0.5$)$. Citrate synthase (Intca_04135), the enzyme catalyzing the conversion of acetyl-CoA 253 to citrate and the first step of the tricarboxylic acid (TCA) cycle, showed a highly significant 254 increase in transcript abundance between EE-LE and -ST (lfc $=4.3 \pm 0.5, p<0.001 ; 6.9 \pm 0.5$, $255 p<0.001)$. 
Within the ETC, there was moderate and significant decrease in transcript abundance for all subunits from the primary dehydrogenase (nио complex; Intca_03465-03539) between EE-LE and -ST (lfc $=-1.2 \pm 0.3 ;-2.4 \pm 0.6, p<0.001)$, respectively. Nitrate reductase subunits showed no change in transcript abundance between EE-LE $(1 \mathrm{fc}=0.01 \pm 0.07)$ and moderately decreased in abundance by ST (lfc $=-1.2 \pm 0.1$ ), which was corroborated by the depletion of nitrate during stationary phase. There was a significant increase in transcript abundance of nirK (Intca_17170) $(1 \mathrm{fc}=2.2 \pm 0.6, p=0.003 ; 2.4 \pm 0.6, p<0.001)$ and quinol dehydrogenase/membrane anchor subunit $n r f H($ Intca_09465) $(\mathrm{lfc}=2.5 \pm 0.6, p=0.001 ; 2.1 \pm 0.6, p=0.003)$ by EE-LT and EE-ST, respectively, which coincided with nitrite production (Figure 3A). The catalytic subunit of the cytochrome c nitrite reductase complex ( $n r f A)$ (Intca_09460) also increased moderately in transcript abundance by EE-LT and EE-ST (lfc $=1.6 \pm 0.6 ; 1.0 \pm 0.6)$, respectively (Figure 3B). Contrary to the transcript abundance patterns of nirK and $n r f A H$, nitric oxide reductase (qNor; Intca_01525) transcripts moderately increased between EE-LT $(1 \mathrm{fc}=1.6 \pm 0.6)$ but decreased in the successive time periods $(1 \mathrm{fc}=0.43 \pm 0.6$ between EE-ST; $1 \mathrm{fc}=-1.2 \pm 0.6$ between LE-ST) (Figure 3B).

271 There was a significant increase in transcript abundance of formate transporter foc $A$

272 (Intca_17150) between EE-ST, as well as LE-ST $(1 \mathrm{fc}=4.9 \pm 0.7, p=0.002 ; 4.8 \pm 0.7, p=0.002$;

273 respectively). We verified the production of formate in our ion chromatography measurements in 274 the range of $100-200 \mu \mathrm{M}$ following late exponential growth. We also observed a moderate 275 increase in transcript abundance of formate dehydrogenase (FDH) subunits (Intca_11150276 11160). These results implicate the activity of formate oxidation, which would contribute to a $\Delta p$ 277 in the periplasm via a Q-loop mechanism and the reduction of MK for electron transfer to nitrite 278 via cytochrome c nitrite reductase. Considering that formate was not provided in our media 279 recipe, an alternative pathway for formate production must exist in I. calvum. We also observed 280 acetate production in similar concentrations as formate $(100-200 \mu \mathrm{M})$. In E. coli, formate is 281 produced anaerobically from the action of pyruvate formate lyase (PFL). We identified a putative 282 PFL based on genome annotation (Intca_12230), where transcript abundance also significantly 283 increased by ST. PFL is also highly sensitive to oxygen [35], which was also in agreement with a 284 significant increase in transcript abundance between EE-ST and LE-ST (Figure 3B) of 285 cytochrome $b d$ oxidase (Intca_01110 and Intca_01115), which is thought to protect anaerobic 286 enzymes against oxidative stress [36]. 


\section{Discussion}

288

289

290

291

292

293

294

295

296

297

298

299

300

301

302

303

304

305

306

307

308

309

310

311

312

313

314

We challenge the paradigm that $\mathrm{C}: \mathrm{NO}_{3}{ }^{-}$ratio controls pathway selection in a dual-pathway organism based on a simple principle: ratios do not account for the abundance of growth-limiting resources. We hypothesized that limitation in $\mathrm{C}_{\text {or }} \mathrm{NO}_{3}{ }^{-}$should better predict pathway selection in a dual-pathway denitrifier/respiratory ammonifier. To test this hypothesis, we systematically measured the response of the Gram-positive Actinobacterium Intrasporangium calvum C5 to the same range of $\mathrm{C}: \mathrm{NO}_{3}{ }^{-}$ratios at both high and low resource loadings to better resolve mechanisms of pathway selection. We demonstrated that resource concentration, not $\mathrm{C}: \mathrm{NO}_{3}{ }^{-}$ratio, influences pathway selection. We found stronger support for respiratory ammonification preference under C-limitation (at low $\mathrm{C}: \mathrm{NO}_{3}{ }^{-}$ratios), which also grew at significantly higher growth rates (Figure 2). These results suggest that the NrfA complex, which receives electrons directly from the MKpool, is optimized to maximize power when one or more resources are limiting. The enrichment of $\mathrm{C}$ and $\mathrm{N}$ ARSC in publically available NrfA over NirK protein seuqences (Figure 1B) provides further support and evolutionary precedence to ammonification preference over denitrification under resource limitation. This is because the end-product of ammonification can be used as an assimilatory N-source (Figure 1C), indicating no evolutionary constraint to cost minimize N. These data, together with metabolic reconstructions from metabolite and transcriptional profiles (Figure 3), suggest that $\mathrm{C}: \mathrm{NO}_{3}{ }^{-}$ratio alone is insufficient to explain pathway selection.

The theoretical basis for pathway selection is explained by the law of the minimum (LM) and the maximum power principle (MPP), which state that growth is limited by the least abundant resource and that biological systems are designed to maximize power in order to effectively allocate energy to reproduction and survival [37,38], respectively. Here, it appears these two theories are working together: when resources are limited, the cell utilizes the respiratory pathway for growth that is optimized to maximize power. Power, in this case, is realized as higher growth rates from the cultures exhibiting disproportionately higher ammonium production than $\mathrm{N}_{2} \mathrm{O}$ production (Figure 2: high resources: $\mathrm{C}: \mathrm{NO}_{3}{ }^{-}$ratio $=0.1$; low resources: $\mathrm{C}: \mathrm{NO}_{3}{ }^{-}$ratios $=4,1,0.5,0.1)$. More specifically, the bacterium must generate a greater $\Delta p$ in order to maximize power when starved for a growth limiting resource. This may help to further explain how respiratory ammonification, which is overall energetically less favorable than denitrification 
317 (lactate with nitrite: $\Delta \mathrm{G}^{\mathrm{o}}=-763.98$ versus $\Delta \mathrm{G}^{\mathrm{o}}=-1196.93$, respectively), can have higher growth yields [39] and growth rates (Figure 2, Figure S1) under C- and N-limitation due to the higher energy yield on a per-nitrite basis (denitrification: -217 KJ per mole nitrite; respiratory ammonification: -399 KJ per mole nitrite). For comparison, a total of $8 \mathrm{H}^{+}$are translocated during denitrification by I. calvum (not including nitrate reduction since both pathways share this step) (Figure 3): NADH dehydrogenase translocates $4 \mathrm{H}^{+}$per $\mathrm{MKH}_{2}$ oxidized and the $\mathrm{bc}_{1}$

323 complex translocates an additional $4 \mathrm{H}^{+}$per $\mathrm{MKH}_{2}$ oxidized. However, $2 \mathrm{H}^{+}$must be consumed 324 in the periplasm to reduce nitrite to $\mathrm{NO}$ [40]. qNor has a net zero $\mathrm{H}^{+}$release (consumes $2 \mathrm{H}^{+}$to make $\mathrm{N}_{2} \mathrm{O}$ but releases $2 \mathrm{H}^{+}$) without $\mathrm{MKH}_{2}$ regeneration [41]. Thus, a net total of $6 \mathrm{H}^{+}$are translocated per nitrite reduced in denitrification with added biosynthetic costs of making the bc $c_{1}$

327 complex and qNor. In respiratory ammonification, $\mathrm{MK} / \mathrm{MKH}_{2}$ redox pair is cycled between 328 NADH dehydrogenase and formate dehydrogenase. 6 electrons and $8 \mathrm{H}^{+}$are needed to reduce 329 nitrite to ammonium, thus $3 \mathrm{MKH}_{2}$ are needed [16]. If $\mathrm{MKH}_{2}$ is received from NADH 330 dehydrogenase, $12 \mathrm{H}^{+}$are translocated plus $2 \mathrm{H}^{+}$from $\mathrm{FDH}$. As each $\mathrm{MKH}_{2}$ is oxidized at the 331 binding site of $\mathrm{NrfH}, 2 \mathrm{H}^{+}$are liberated [16], resulting in a net total of $12 \mathrm{H}^{+}$translocated per 332 nitrite reduced for respiratory ammonification. This implies that the cell might deplete its NADH 333 pool more rapidly on a per nitrite basis. However, if more protons are pumped in the early stages 334 of growth, the cell would be allocating the ATP generated for anabolism, as evidenced by higher 335 growth rates in the cultures exhibiting higher amounts of respiratory ammonification (Figure 2), 336 which is supported by the MPP.

337 Under our high resource conditions (Figure 2; left panels), at $\mathrm{C}: \mathrm{NO}_{3}{ }^{-}$ratios $\geq 1$, we observed that denitrification prevailed and these cultures had lower growth rates than the predominantly ammonium producing cultures. These high resource circumstances resulted in the production of

341 why these cultures had lower growth rates (Figure 2; left panels) and quasi-steady state growth 342 curves in our high resource metabolite profile (Figure 3A). Rowley and colleagues [42] reported 343 that at least $20 \%$ of the $\mathrm{N}_{2} \mathrm{O}$ released during high $\mathrm{C}$ conditions were produced by competition 344 between nitrite and nitrate in the active-site of NarG. Under excess C concentrations, NarG 345 produces intracellular $\mathrm{NO}$ from $\mathrm{NO}_{2}{ }^{-}$and these intermediates are likely inhibitory to cell growth, 346 which may explain why our growth curves (Figure 3A) reached a quasi-steady state before 347 nitrate had been fully utilized (as compared to the low resource metabolite profile, Figure S2). 
348 Furthermore, resources were not limiting growth under these conditions. Rather, the cells were

349 likely experiencing toxicity from $\mathrm{NO}$ and $\mathrm{NO}_{2}{ }^{-}$and thus the metabolic outcomes would be beyond the scope of the LM and MPP. Nonetheless, these results clearly demonstrate that endproduct formation from the two resource concentrations tested, with the same $\mathrm{C}: \mathrm{NO}_{3}{ }^{-}$ratios, are not identical thereby refuting the $\mathrm{C}: \mathrm{NO}_{3}{ }^{-}$control hypothesis.

353 We selected a single treatment ( $8 \mathrm{mM}$ lactate, $12 \mathrm{mM}$ nitrate; $\mathrm{C}: \mathrm{NO}_{3}{ }^{-}$ratio $\left.=2\right)$, in which we observed both denitrification and respiratory ammonification occurring simultaneously, for RNA-Seq in order to gain insight into the transcriptional organization of actively growing $I$.

356 calvum cells (Figure 3). Strangely, we saw a decrease in transcript abundance encoding for two 357 enzymes known to convert lactate to pyruvate, LDH and LUP. While normalized read counts 358 (Figure S5) were generally consistent across growth phases, indicative of constitutive expression, 359 further research investigating the mode of anaerobic lactate oxidation in I. calvum would 360 illuminate how reducing equivalents are fed into its central metabolic pathway. For example, $S$. 361 loihica PV-4 is known to use lactate for both denitrification and respiratory ammonification, but 362 only uses acetate for denitrification [24]. Nonetheless, our transcriptomic data suggests that 363 pyruvate plays a central role in providing reducing equivalents to the TCA cycle as Acetyl-CoA, 364 as evidenced by significant upregulation in the genes encoding for pyruvate dehydrogenase and 365 citrate synthase, as well as apparent "leaking" via incomplete lactate oxidation through the 366 release of acetate and formate. Such leaking may be produced by a putative PFL, adding to the 367 diversity of C utilization pathways feeding the ETC, and thereby driving pathway selection for 368 nitrite reduction. Our transcriptomic results, coupled with a parallel metabolite profile (Figure 3), 369 also suggest that the dual-pathway is induced by the presence of nitrite, and is not constitutively 370 expressed like nitrate reductase, $n a r G$. Furthermore, it appears that the significant increase in 371 transcript abundance for the gene encoding the $b d$ oxidase helps to protect the anaerobic372 dependent biochemical machinery against oxidative stress, thereby scavenging any residual 373 oxygen during anaerobic growth.

374 Our metabolite profiles for $\mathrm{N}$ oxyanion respiration and $\mathrm{N}_{2} \mathrm{O}$ versus ammonium production show 375 conflicting patterns relative to previous studies (Figure 3A, Figure S2). Yoon and colleagues [43] 376 reported complete reduction of nitrate, production of nitrite, and then rapid consumption of 377 nitrite, with $\mathrm{N}_{2} \mathrm{O}$ as the main end-product, by $S$. loihica PV-4 (5 mM lactate, $1 \mathrm{mM}$ nitrate; 
ratio=0.6). When Yoon and colleagues [43] replaced nitrate with nitrite as the dominant electron acceptor (5 mM lactate, $1 \mathrm{mM}$ nitrite, ratio=0.6), ammonification prevailed. Other research has shown the same response to nitrite replacement and ammonification dominance using nonfermentable C-sources (i.e., acetate) in chemostat enrichments of Geobacter lovleyi[44]. In our work, nitrite was never fully depleted (Figure 3A, Figure S2, Figure S3) and when nitrite was given as the only electron acceptor, the bacterium predominantly used denitrification but without concurrent growth (Figure S4). Similar to our work, Kraft and colleagues[34] also reported denitrification dominance when nitrite was supplied as the terminal acceptor. These differences highlight an incomplete understanding for the molecular mechanisms underlying the framework put forth by the LM and MPP.

A detailed look into the biochemistry of ETC complexes helps to shed light on the molecular mechanisms modulating pathway bifurcation. For example, Yoon and colleagues [3] demonstrated that elevated $\mathrm{pH}$ selects for ammonification in S. loihica PV-4. This phenotypic response is due to a decrease in the midpoint potential of the Rieske protein at higher $\mathrm{pH}$ [4548]. Thus, any hindrance of electron flow through the $b_{1}$ complex would ultimately reduce the activity of downstream processes and promote alternative respiratory pathways. Nitrogen and C limitation have also been shown to influence flux distributions in redox sensitive proteins, including those found in electron transport [49]. A drop in the intracellular redox potential (redox poise) of the cell due to resource limitation may decrease the midpoint potential of the Rieske protein and reduce the activity of any downstream electron exit modules, such as NirK [50-52]. Thus, based on fundamental principles of protein redox chemistry and thermodynamics, it becomes clear that denitrification versus ammonification are likely not modulated by an arbitrary ratio of $\mathrm{C}: \mathrm{NO}_{3}{ }^{-}$, but rather by thermodynamic constraints of the Q-cycle $[11,12]$. The phenotypic response of higher rates of denitrification over ammonification at high $\mathrm{C}: \mathrm{NO}_{3}{ }^{-}$ratios in other published studies $[3,4]$ may also be due to enrichment bias for organisms that utilize quinones with higher midpoint potentials in their bioenergetic chains (Figure 1). Bergdoll and colleagues [11] suggested that comparisons of Rieske/cyt $b$ complexes from organisms with high- and lowpotential quinones may help to reconcile the thermodynamic properties of Q-cycle function. 
calvum uses a MK-based LP bioenergetic chain it may be possible that the differences in pathway selection across treatments are unique to LP chains.

410 Piecing together the evolutionary history of the N-cycle using isotopic signatures for 411 geochemically available $\mathrm{N}$ module cofactors (i.e., Ni, Fe, and Mo) coupled to molecular

412 evolutionary analysis has revealed respiratory ammonification was likely a major component of

413 the Archean N-cycle [33]. Abiotic nitrite formation and depletion of ammonia through

414 photodissociation [53] would have created selective pressures for a dissimilatory N pathway that 415 also produced assimilatory N. We demonstrate that NrfA proteins are significantly enriched in N 416 compared to NirK (i.e., no evolutionary constraints to cost minimize $\mathrm{N}$ in the $n r f A$ gene product 417 [15]) (Figure 1B) and that ammonium production (without accumulation in the medium) 418 supports growth in I. calvum (Figure 1C). The Nrf module is also relatively simplistic in that it 419 receives electrons directly from the quinol pool and not the $b_{1} c_{1}$ complex used in denitrification.

420 The early exit of electrons from the ETC (i.e., before reaching the bc ${ }_{1}$ complex) suggests that Nrf 421 may have originated prior to the $b_{1}$ complex. Furthermore, the quinol oxidation site $\left(Q_{0}\right)$ of 422 cytochrome $b$ contains a PDWY motif, indicative of an ancestral LP respiratory chain found in 423 many Gram-positive organisms [54]. However, there is still debate regarding the presence of a 424 cytochrome $b c$ complex in the last universal common ancestor [54, 55]. Lastly, the Nrf module is 425 wired to operate via a q-loop with formate dehydrogenase whose Mo-cofactors would have also 426 been bioavailable during the Archean, further supporting an early evolution.

427 In summary, we employ a new predictive framework that accounts for the biochemistry and 428 evolutionary history of $\mathrm{N}$ modules, ETC complexes, and pool quinones to suggest the 429 mechanisms by which these two pathways are regulated at the molecular level. With this 430 understanding, it may be possible to extend our framework to environmental microbial 431 populations and accelerate model development across different ecosystem scales (i.e., cross-scale 432 systems biology).

ACKNOWLEDGMENTS. We thank F. von Netzer, K. Hunt, S Morales, N. Stopnisek, K. Meinhardt, W. Qin, N Elliot, T. Hazen, H. Carlson, B. Ramsey, A. Murray, Z. Harrold, T. Morgan, and P. Longley for thoughtful feedback and discussions. This research was supported by a grant from the Nevada Governor's Office of Economic Development (JG), by the Desert Research Institute (DRI) postdoctoral research fellowship program, and in part by

437 Ecosystems and Networks Integrated with Genes and Molecular Assemblies (ENIGMA) (http://enigma.lbl.gov) —a

438 Scientific Focus Area Program at Lawrence Berkeley National Laboratory under contract number DE-AC02- 
05CH11231 and funded in part by Oak Ridge National Laboratory under contract DE-AC05-00OR22725, and is based upon work supported by the U.S. Department of Energy, Office of Science, Office of Biological \& Environmental Research.

\section{Competing Interests}

\section{The authors declare no conflicts of interest}

\section{References}

445 1. Knowles R. Denitrification. Microbiol Rev 1982; 46: 43-70.

446 2. Tiedje JM, Sexstone AJ, Myrold DD, Robinson JA. Denitrification: ecological niches , competition and 447 survival. Antonie Van Leeuwenhoek 1982; 48: 569-583.

448 3. Yoon S, Cruz-García C, Sanford R, Ritalahti KM, Löffler FE. Denitrification versus respiratory

4. van den Berg EM, van Dongen U, Abbas B, van Loosdrecht MC. Enrichment of DNRA bacteria in a ammonification: Environmental controls of two competing dissimilatory NO 3-/NO 2-reduction pathways in Shewanella loihica strain PV-4. ISME J 2015; 9: 1093-1104.

453 5. Schmidt CS, Richardson DJ, Baggs EM. Constraining the conditions conducive to dissimilatory nitrate

6. Hardison AK, Algar CK, Giblin AE, Rich JJ. Influence of organic carbon and nitrate loading on partitioning between dissimilatory nitrate reduction to ammonium (DNRA) and N2 production. Geochim Cosmochim Acta 2015; 164: 146-160.

7. Fazzolari É, Nicolardot B, Germon JC. Simultaneous effects of increasing levels of glucose and oxygen

8. Galloway JN, Aber JD, Erisman JW, Seitzinger SP, Howarth RW, Cowling EB, et al. The Nitrogen partial pressures on denitrification and dissimilatory nitrate reduction to ammonium in repacked soil cores. Eur J Soil Biol 1998; 34: 47-52.

9. Moparthi VK, Hägerhäll C. The evolution of respiratory chain complex i from a smaller last common

10. Dibrova D V., Cherepanov DA, Galperin MY, Skulachev VP, Mulkidjanian AY. Evolution of cytochrome ancestor consisting of 11 protein subunits. J Mol Evol 2011; 72: 484-497.

470 12. Schoepp-Cothenet B, Lieutaud C, Baymann F, Verméglio A, Friedrich T, Kramer DM, et al. Menaquinone bc complexes: From membrane-anchored dehydrogenases of ancient bacteria to triggers of apoptosis in vertebrates. Biochim Biophys Acta - Bioenerg 2013; 1827: 1407-1427.

13. Soo RM, Hemp J, Parks DH, Fischer WW, Hugenholtz P. On the origins of oxygenic photosynthesis and aerobic respiration in Cyanobacteria. Science (80- ) 2017; 355: 1436-1440.

11. Bergdoll L, Ten Brink F, Nitschke W, Picot D, Baymann F. From low- to high-potential bioenergetic chains: Thermodynamic constraints of Q-cycle function. Biochim Biophys Acta 2016; 1857: 1569-1579. as pool quinone in a purple bacterium. Proc Natl Acad Sci 2009; 106: 8549-54.

Baudouin-Cornu P, Schuerer K, Marlière P, Thomas D. Intimate evolution of proteins: Proteome atomic content correlates with genome base composition. J Biol Chem 2004; 279: 5421-5428. 
15. Grzymski JJ, Dussaq AM. The significance of nitrogen cost minimization in proteomes of marine microorganisms. ISME J 2012; 6: 71-80.

16. Einsle O, Messerschmidt A, Huber R, Kroneck PMH, Neese F. Mechanism of the six-electron reduction of nitrite to ammonia by cytochrome c nitrite reductase. J Am Chem Soc 2002; 124: 11737-11745.

17. Kraft B, Strous M, Tegetmeyer HE. Microbial nitrate respiration - Genes, enzymes and environmental distribution. J Biotechnol 2011; 155: 104-117.

18. Godfrey L V., Falkowski PG. The cycling and redox state of nitrogen in the Archaean ocean. Nat Geosci 2009; $2: 725-729$.

19. Nitschke W, Dracheva S. Reaction center associated cytochromes in Anoxygenic Photosynthetic Bactera. In: Blankenship R, Madigan M, Bauer C (eds).1995. Kluwer Academic, Dordrecht, The Netherlands, pp $775-805$.

20. Lalucat J, Bennasar A, Bosch R, Garcia-Valdes E, Palleroni NJ. Biology of Pseudomonas stutzeri. Microbiol Mol Biol Rev 2006; 70: 510-547.

21. Suharti, de Vries S. Membrane-bound denitrification in the Gram-positive bacterium Bacillus azotoformans. Biochem Soc Trans 2005; 33: 130-133.

491 22. Heylen K, Keltjens J, Stein LY. Redundancy and modularity in membrane-associated dissimilatory nitrate reduction in Bacillus. Front Microbiol 2012; 3: 1-27.

Decleyre H, Heylen K, Bjorn T, Willems A. Highly diverse nirK genes comprise two major clades that harbour ammonium-producing denitrifiers. BMC Genomics 2016; 17: 1-13.

24. Yoon S, Sanford RA, Löffler FE. Shewanella spp. Use acetate as an electron donor for denitrification but not ferric iron or fumarate reduction. Appl Environ Microbiol 2013; 79: 2818-2822.

25. Hillesland KL, Lim S, Flowers JJ, Turkarslan S, Pinel N, Zane GM, et al. Erosion of functional independence early in the evolution of a microbial mutualism. Proc Natl Acad Sci 2014; 111: 14822-14827. Health Association: Washington, DC.

501 27. Koren S, Walenz BP, Berlin K, Miller JR, Bergman NH, Phillippy AM. Canu : scalable and accurate long- - read assembly via adaptive k - - - mer weighting and repeat separation. Genome Res 2017; 1-35. Sequence With Rearrangements. Genome Res 2004; 14: 1394-1403.

Darling AE, Mau B, Perna NT. Progressivemauve: Multiple genome alignment with gene gain, loss and rearrangement. PLoS One 2010; 5: e11147.

507 30. Andrews S. FastQC: A quality control tool for high throughput sequence data. Available online at: http:// www.bioinformatics.babraham.ac.uk/projects/fastqc 2010.

509 31. Bolger AM, Lohse M, Usadel B. Trimmomatic: A flexible trimmer for Illumina sequence data.

$510 \quad$ Bioinformatics 2014; 30: 2114-2120.

511 32. Kalakoutskii LV, Kirillova IP, Krassilnikov NA. a New Genus of the. J Gen Microbiol 1967; 48: 373-79-

51285.

513 33. Klotz MG, Stein LY. Nitrifier genomics and evolution of the nitrogen cycle. FEMS Microbiol Lett 2008; 
34. Kraft B, Tegetmeyer HE, Sharma R, Klotz MG, Ferdelman TG, Hettich RL, et al. The environmental controls that govern the end product of bacterial nitrate respiration. Science (80- ) 2014; 345: 676-679.

35. Abbe KS, Takahashi S, Yamada T. Invovlement of oxygen-sensitive pyruvate-formate lysase in mixed-acid fermetnation by Streptococcus mutans under strictly anerobic conditions. J Bacteriol 1982; 152: 175.

Das A, Silaghi-Dumitrescu R, Ljungdahl LG, Kurtz DM. Cytochrome $b d$ oxidase, oxidative stress, and dioxygen tolerance of the strictly anaerobic bacterium Moorella thermoacetica. J Bacteriol 2005; 187: 2020-2029.

522 37. Lotka AJ. Contribution to the Energetics of Evolution. Proc Natl Acad Sci 1922; 8: 147-151.

523 38. DeLong JP. The maximum power principle predicts the outcomes of two-species competition experiments.

39. Strohm TO, Griffin B, Zumft WG, Schink B. Growth yields in bacterial denitrification and nitrate Oikos 2008; 117: 1329-1336. ammonification. Appl Environ Microbiol 2007; 73: 1420-1424.

42. Rowley G, Hensen D, Felgate H, Arkenberg A, Appia-ayme C, Prior K, et al. Resolving the contributions of the membrane-bound and periplasmic nitrate reductase systems to nitric oxide and nitrous oxide production in Salmonella enterica serovar Typhimurium. Biochem J 2012; 762: 755-762. Structural Modeling. In: Schäfer G, Penefsky HS (eds). Results and Problems in Cell Differentiation. 2008. Springer Science+Business Media, pp 1-28.

43. Yoon S, Sanford RA, Löffler FE. Nitrite control over dissimilatory nitrate/nitrite reduction pathways in Shewanella loihica strain PV-4. Appl Environ Microbiol 2015; 81: 3510-3517.

44. van den Berg EM, Rombouts JL, Kuenen JG, Kleerebezem R, van Loosdrecht MCM. Role of nitrite in the competition between denitrification and DNRA in a chemostat enrichment culture. AMB Express 2017; 7: $1-7$.

45. Link TA, Hagen WR, Pierik AJ, Assmann C, von Jagow G. Determination of the redox properties of the Rieske cluster of bovine heart bc 1 complex by direct electrochemistry of a water- soluble fragment. EurJBiochem 1992; 208: 685-691.

46. Ugulava NB, Crofts AR. CD-monitored redox titration of the Rieske Fe-S protein of Rhodobacter sphaeroides: $\mathrm{pH}$ dependence of the midpoint potential in isolated bc 1 complex and in membranes. FEBS Lett 1998; 440: 409-413.

47. Zu Y, Couture MMJ, Kolling DRJ, Crofts AR, Eltis LD, Fee JA, et al. Reduction Potentials of Rieske 2003; 42: 12400-12408.

48. Trumpower BL. Cytochrome bc1 complexes of microorganisms. Microbiol Rev 1990; 54: 101-129.

49. Ansong C, Sadler NC, Hill EA, Lewis MP, Zink EM, Smith RD, et al. Protein redox dynamics during lightto-dark transitions in cyanobacteria and impacts due to nutrient limitation. Front Microbiol 2014; 5: 1-10.

50. Snyder CH, Merbitz-Zahradnik T, Link TA, Trumpower BL. Role of the Rieske Iron - Sulfur Protein Midpoint Potential in the Protonmotive Q-Cycle Mechanism of the Cytochrome bc 1 Complex. J Bioenerg Biomembr 1999; 31: 235-242.

51. Denke E, Merbitz-Zahradnik T, Hatzfeld OM, Snyder CH, Link TA, Trumpower BL. Alteration of the midpoint potential and catalytic activity of the Rieske iron-sulfur protein by changes of amino acids forming 
hydrogen bonds to the iron-sulfur cluster. J Biol Chem 1998; 273: 9085-9093.

52. Hunte C, Solmaz S, Palsdóttir H, Wenz T. A structural perspective on mechanism and function of the cytochrome bc1 complex. In: Schäfer G, Penefsky HS (eds). Results and Problems in Cell Differentiation. 2008. Springer Science+Business Media, pp 253-270.

53. Falkowski PG. Evolution of the nitrogen cycle and its influence on the biological sequestration of CO2 in the ocean. Nature 1997; 387: 272-275.

54. Kao WC, Hunte C. The molecular evolution of the Qo Motif. Genome Biol Evol 2014; 6: 1894-1910.

55. Dibrova D V., Cherepanov DA, Galperin MY, Skulachev VP, Mulkidjanian AY. Evolution of cytochrome bc complexes: From membrane-anchored dehydrogenases of ancient bacteria to triggers of apoptosis in vertebrates. Biochim Biophys Acta - Bioenerg 2013; 1827: 1407-1427.

56. White D. The physiology and biochemistry of prokaryotes, 3rd ed. 2000. Oxford University Press.

57. Thauer RK, Jungermann K, Decker K. Energy conservation in chemotrophic anaerobic bacteria. Bacteriol Rev 1977; 41: 100-180.

58. Giardina CP, Ryan MG. Total belowground carbon allocation in a fast-growing Eucalyptus plantation estimated using a carbon balance approach. Ecosystems 2002; 5: 487-499.

59. Edgar RC. MUSCLE: multiple sequence alignment with high accuracy and high throughput. Nucleic Acids Res 2004; 32: 1792-7.

60. Tamura K, Peterson D, Peterson N, Stecher G, Nei M, Kumar S. MEGA5: Molecular evolutionary genetics analysis using maximum likelihood, evolutionary distance, and maximum parsimony methods. Mol Biol Evol 2011; 28: 2731-2739.

61. Stamatakis A. RAxML version 8: A tool for phylogenetic analysis and post-analysis of large phylogenies. Bioinformatics 2014; 30: 1312-1313.

62. Kelley LA, Mezulis S, Yates C, Wass M, Sternberg M. The Phyre2 web portal for protein modelling, prediction, and analysis. Nat Protoc 2015; 10: 845-858.

63. Dobin A, Davis CA, Schlesinger F, Drenkow J, Zaleski C, Jha S, et al. STAR: Ultrafast universal RNA-seq aligner. Bioinformatics 2013; 29: 15-21.

64. Liao Y, Smyth GK, Shi W. FeatureCounts: An efficient general purpose program for assigning sequence reads to genomic features. Bioinformatics 2014; 30: 923-930.

65. Love MI, Huber W, Anders S. Moderated estimation of fold change and dispersion for RNA-seq data with DESeq2. Genome Biol 2014; 15: 1-21. 


\section{Figure legends}

Figure 1. (A) Maximum likelihood phylogenetic tree of NrfA amino acid sequences from known respiratory ammonifiers and accompanying N-module composition for each organism. Pool quinone is also noted for dual-pathway nitrite reducers and model species. Colors of the main branches denote the $1^{\text {st }}$ heme motif type: $\mathrm{CxxCK}$ and $\mathrm{CxxCH}$. (B) Protein atomic composition for $\mathrm{N}$ and $\mathrm{C}$ normalized to protein length for NirK and NrfA nitrite reductases. (C) Statetransition from ammonium-replete to ammonium-deplete for I. calvum $\mathrm{C} 5$ grown under $8 \mathrm{mM}$ lactate $12 \mathrm{mM}$ nitrate minimal media at $30^{\circ} \mathrm{C}$. Metabolite profiles for ammonium-deplete are shown.

Figure 2. The effects of high resource (left; range of lactate concentrations with $12 \mathrm{mM} \mathrm{NO}_{3}{ }^{-}$) and low resource (right; range of lactate concentrations with $1.2 \mathrm{mM} \mathrm{NO}_{3}{ }^{-}$) concentrations with the same C: $\mathrm{NO}_{3}{ }^{-}$ratio on pathway selection in I. calvum C5. (A) Production of $\mathrm{N}_{2} \mathrm{O}-\mathrm{N}$ and net change of $\mathrm{NH}_{4}{ }^{+}$over a 100 -hour incubation period at $30^{\circ} \mathrm{C}$. Each bar represents the average of 8-10 replicates per treatment (Table S5). (B) Fraction of dissimilated $\mathrm{N}$ by pathway. (C) Growth rates for each corresponding treatment. The x-axis label defines lactate concentration and $\mathrm{C}: \mathrm{NO}_{3}{ }^{-}$ ratio in parentheses.

Figure 3. (A) Time-series metabolite profiles for lactate, nitrate, and nitrite (top pane), production of dissimilated end-products as $\mathrm{N}_{2} \mathrm{O}-\mathrm{N}$ and net change in $\mathrm{NH}_{4}{ }^{+}$ammonium production (middle pane), and corresponding growth curve of I. calvum cells grown under $8 \mathrm{mM}$ lactate $12 \mathrm{mM}$ nitrate $\left(\mathrm{C}: \mathrm{NO}_{3}{ }^{-}\right.$ratio $\left.=2\right)$ (bottom pane). Sampling points during growth phases are marked where transcriptomic profiling was performed (red arrows). (B) Metabolic reconstruction of the ETC from I. calvum with transcriptional changes for genes participating in dual-pathway dissimilatory nitrite reduction. $\log _{2}$ fold changes in transcript abundance are shown for late exponential relative to early exponential growth phase (EE vs. LE), stationary phase relative to early exponential growth phase (EE vs. ST), and stationary phase relative to late exponential growth phase (LE vs. ST). Locus IDs for each gene product correspond to heat map subplots in the order shown (left-to-right for each growth phase and top-to-bottom for each locus ID specified). Higher transcript abundance is represented in red, lower transcript abundance in blue, and no change in transcript abundance in white. Significant changes in transcript abundance $(p<0.01)$ are marked as a red box. Value of $\log _{2}$ fold change is specified within each subplot. The $\log _{2}$ fold changes of 14 NADH dehydrogenase subunits (Intca_03465-03530) were averaged as transcriptional changes were all shifted in the same direction. 

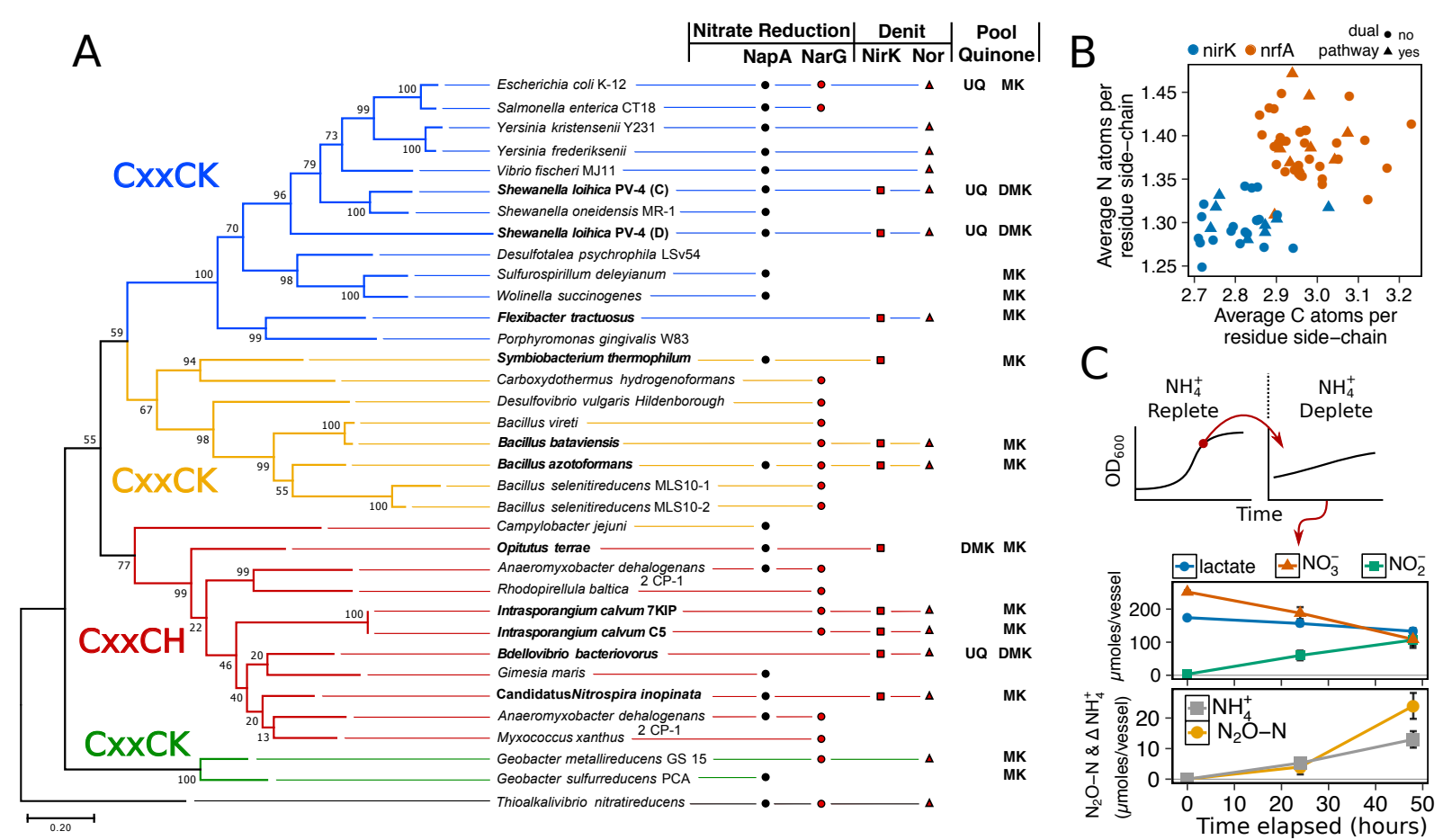

Figure 1. (A) Maximum likelihood phylogenetic tree of NrfA amino acid sequences from known respiratory ammonifiers and accompanying N-module composition for each organism. Pool quinone is also noted for dual-pathway nitrite reducers and model species. Colors of the main branches denote the $1^{\text {st }}$ heme motif type: $\mathrm{CxxCK}$ and $\mathrm{CxxCH}$. (B) Protein atomic composition for $\mathrm{N}$ and $\mathrm{C}$ normalized to protein length for NirK and NrfA nitrite reductases. (C) Statetransition from ammonium-replete to ammonium-deplete for I. calvum $\mathrm{C} 5$ grown under $8 \mathrm{mM}$ lactate $12 \mathrm{mM}$ nitrate minimal media at $30^{\circ} \mathrm{C}$. Metabolite profiles for ammonium-deplete are shown. 


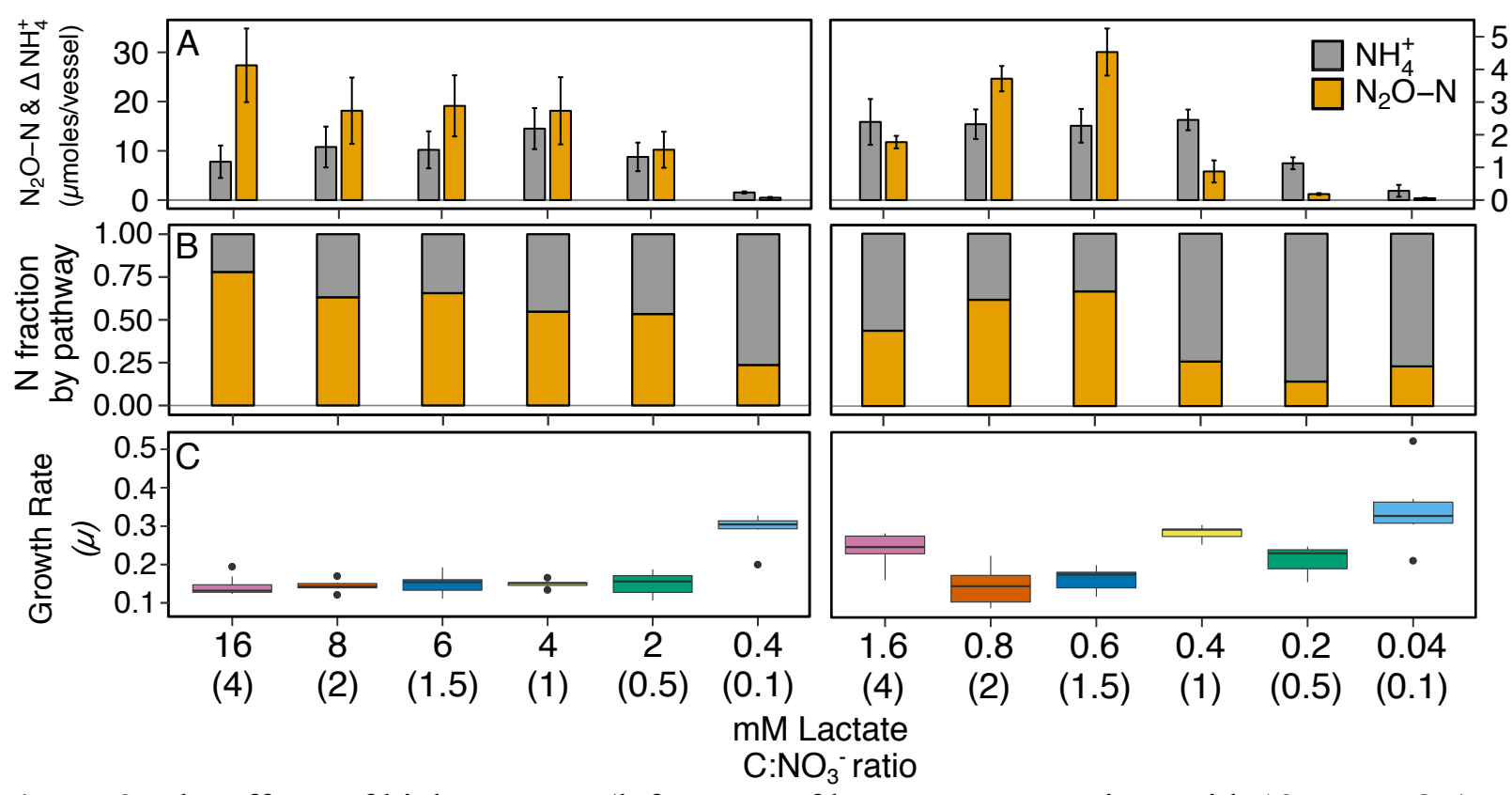

Figure 2. The effects of high resource (left; range of lactate concentrations with $12 \mathrm{mM} \mathrm{NO}_{3}{ }^{-}$) and low resource (right; range of lactate concentrations with $1.2 \mathrm{mM} \mathrm{NO}_{3}{ }^{-}$) concentrations with the same $\mathrm{C}: \mathrm{NO}_{3}{ }^{-}$ratio on pathway selection in I. calvum C5. (A) Production of $\mathrm{N}_{2} \mathrm{O}-\mathrm{N}$ and net change of $\mathrm{NH}_{4}{ }^{+}$over a 100 -hour incubation period at $30^{\circ} \mathrm{C}$. Each bar represents the average of 8-10 replicates per treatment (Table S5). (B) Fraction of dissimilated $\mathrm{N}$ by pathway. (C) Growth rates for each corresponding treatment. The $\mathrm{x}$-axis label defines lactate concentration and $\mathrm{C} \mathrm{NO}_{3}{ }^{-}$ ratio in parentheses. 


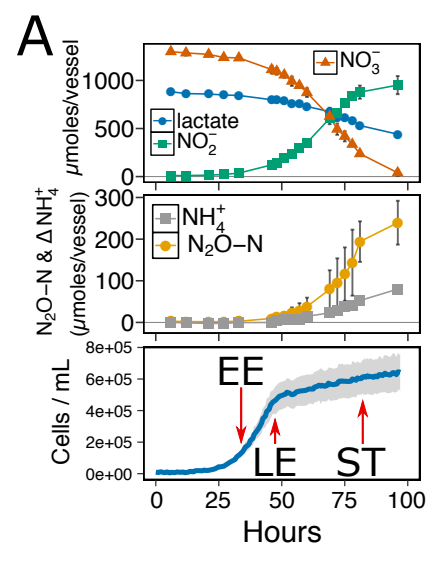

B
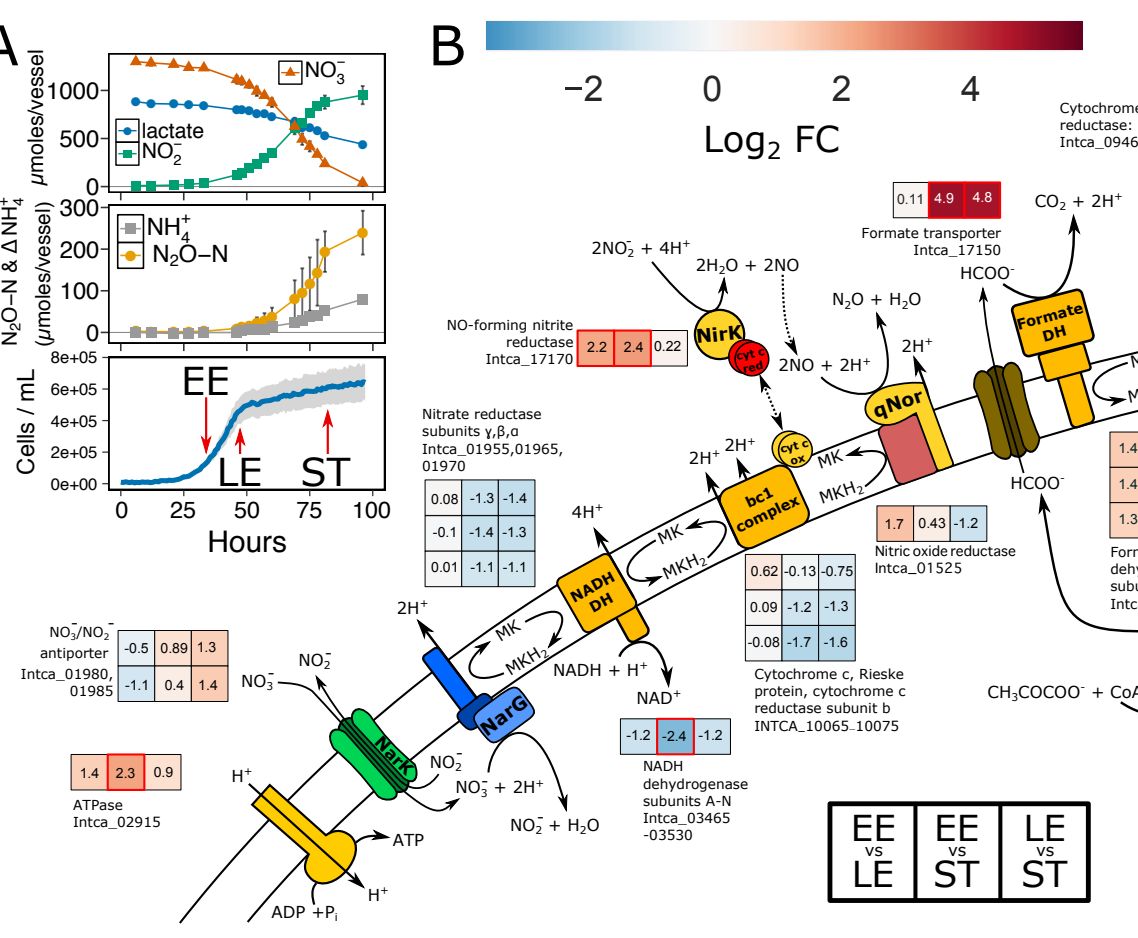

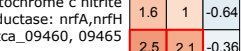

\begin{tabular}{llll} 
Intca_ $-09460,09465$ & 2.5 & 2.1 & -0.36 \\
\hline
\end{tabular}

Figure 3. (A) Time-series metabolite profiles for lactate, nitrate, and nitrite (top pane), production of dissimilated end-products as $\mathrm{N}_{2} \mathrm{O}-\mathrm{N}$ and net change in $\mathrm{NH}_{4}{ }^{+}$ammonium production (middle pane), and corresponding growth curve of I. calvum cells grown under 8mM lactate $12 \mathrm{mM}$ nitrate $\left(\mathrm{C}: \mathrm{NO}_{3}{ }^{-}\right.$ratio $\left.=2\right)$ (bottom pane). Sampling points during growth phases are marked for transcriptomic analysis. (B) Metabolic reconstruction of the ETC from I. calvum with transcriptional changes for genes participating in dual-pathway dissimilatory nitrite reduction. $\log _{2}$ fold changes in transcript abundance are shown for late exponential relative to early exponential growth phase (EE vs. LE), stationary phase relative to early exponential growth phase (EE vs. ST), and stationary phase relative to late exponential growth phase (LE vs. ST). Locus IDs for each gene product correspond to heat map subplots in the order shown (leftto-right for each growth phase and top-to-bottom for each locus ID specified). Higher transcript abundance is represented in red, lower transcript abundance in blue, and no change in transcript abundance in white. Significant changes in transcript abundance $(p<0.01)$ are marked as a red box. Value of $\log _{2}$ fold change is specified within each subplot. The $\log _{2}$ fold changes of 14 NADH dehydrogenase subunits (Intca_03465-03530) were averaged as transcriptional changes were all shifted in the same direction. 